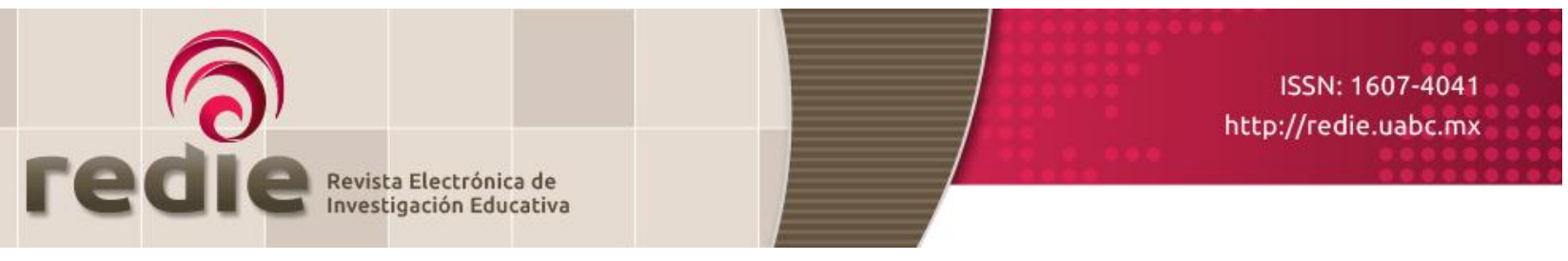

Vol. 20, Núm. 2, 2018

\title{
Violencia escolar en Michoacán y Nuevo León, un diagnóstico situado para promover escuelas seguras en educación básica
}

\section{School Violence in Michoacán and Nuevo León, a Situated Diagnosis for Promoting Safe Schools in Basic-Level Education}

\author{
Sergio Reyes-Angona (*) sergiosofo@gmail.com \\ Sandra Gudiño Paredes $\left(^{*}\right)$ sandra.gudino@itesm.mx \\ Juan Manuel Fernández-Cárdenas (*) j.m.fernandez@itesm.mx \\ (*) Tecnológico de Monterrey \\ (Recibido: 30 de agosto de 2016; Aceptado para su publicación: 7 de febrero de 2017)
}

Cómo citar: Reyes-Angona, S., Gudiño, S. y Fernández-Cárdenas, J. M. (2018). Violencia escolar en Michoacán y Nuevo León, un diagnóstico situado para promover escuelas seguras en educación básica. Revista Electrónica de Investigación Educativa, 20(2), $46-58$. https://doi.org/10.24320/redie.2018.20.2.1548

\section{Resumen}

La complejidad del fenómeno de la violencia escolar requiere programas orientados a mejorar de manera integral el ambiente de convivencia. En este estudio se presenta un diagnóstico exploratorio de la convivencia en diez escuelas de dos estados de la República Mexicana: Michoacán y Nuevo León. Se combina el análisis cuantitativo de un cuestionario diagnóstico de la violencia aplicado a 1,950 alumnos con el análisis cualitativo de 50 entrevistas a profundidad realizadas a alumnos, docentes y directores de los planteles. Los resultados permiten entender el fenómeno de forma situada, reconocer formas de violencia no visibilizadas y fundamentar modelos de intervención futuros bajo una orientación más participativa.

Palabras clave: Convivencia escolar, moral, educación dialógica.

\section{Abstract}

The complexity of the phenomenon of violence in schools calls for programs oriented toward a comprehensive improvement of the living environment. This study presents an exploratory diagnosis of coexistence in ten schools in two states of the Mexican Republic: Michoacán and Nuevo León. A quantitative analysis of a diagnostic questionnaire on violence administered to 1,950 students is combined with a qualitative analysis of 50 in-depth interviews with students, teachers, and school directors. The results make it possible to understand the phenomenon in a situated manner, recognize invisible forms of violence, and inform models for future intervention based on a more participatory approach.

Keywords: School life, moral, dialogic education. 


\section{Introducción}

La iniciativa gubernamental de mayor alcance en México para combatir la violencia en las escuelas en los últimos años (programa nacional de Escuela Segura) concluyó su período de aplicación. Su principal línea de acción, de acuerdo a Fierro (2008), fue dotar a las escuelas de material educativo, actividades y asesoría para crear una cultura de la seguridad, así como impulsar una agenda de investigación sobre el ambiente escolar.

La evaluación y discusión de sus resultados es estratégica para definir en México las políticas públicas de convivencia escolar en los próximos años. Una de las críticas al programa es, precisamente, la falta de indicadores sólidos para evaluar su impacto (Auditoría Superior de la Federación, 2013). En el análisis FODA señala la débil instrumentación de los apoyos financieros, así como la falta de diagnósticos locales que permitan una mejor comprensión del fenómeno e implementación del programa (Zorrilla, 2008). No obstante, los informes estatales anuales impulsados por la Secretaría de Educación Pública (SEP, 2015) ofrecen un panorama detallado de la convivencia escolar a partir de cuestionarios aplicados a los diferentes agentes escolares de un número muy amplio de escuelas.

A pesar de la utilidad de esos datos, que contribuyen a dimensionar el problema, el mapa que presentan sobre la convivencia escolar es incompleto; estudios como los de Klein, Cornell y Konold (2012) y Nickerson, Singleton, Schnurr y Collen (2014) defienden la necesidad de conocer el ambiente de convivencia desde el interior de las instituciones escolares. En México, el estado de conocimiento sobre convivencia, disciplina y violencia escolar recopilado por Furlán y Spitzer (2013) revela las particularidades que adopta el fenómeno en cada región y contexto educativo y contribuye a problematizar la mitificación de la escuela como entorno de protección frente a un entorno exterior socialmente hostil.

A pesar del impulso dado en México a una agenda legislativa ambiciosa para la protección de los derechos de los niños en las escuelas (Zurita, 2016), la perspectiva prescriptiva-normativa no genera por sí sola el impacto deseado -como afirman Fierro, Lizardi, Tapia y Juárez (2013). Estudios realizados en escuelas secundarias, como el de Gómez-Nashiki (2014) en Colima y el de Vega y González (2016) en Guadalajara, confirman la percepción que maestros y estudiantes tienen de las escuelas: escenarios que no disuaden en la práctica los comportamientos agresivos.

Entendemos la violencia escolar, por tanto, como un fenómeno "construido por los actores a través de procesos de interpretación [...] dentro de prácticas socioculturales locales" (Furlán y Saucedo, 2008, p. 237). En consecuencia, construir un buen ambiente de convivencia trasciende la mejor aplicación de regulaciones punitivas y políticas orientadas a la protección física. Como afirman Ortega, Ramírez y Castelán (2005, p.164): "Las intervenciones que han logrado mejores resultados involucran a toda la escuela y a su comunidad".

Por ello es necesario disponer de diagnósticos del efecto de programas gubernamentales de escala nacional (como ES) a partir de investigaciones que: a) sean situadas, de manera tal que permitan apreciar la influencia del entorno local y social, y b) que combinen la representatividad de los datos obtenidos desde una evaluación externa con la introspección desde la perspectiva de los sujetos afectados de esas comunidades.

Esta investigación busca dar respuesta a la pregunta: ¿Cómo es el ambiente de convivencia que prevalece al interior de los centros educativos de nivel secundaria beneficiados por el programa Escuela Segura (ES) desde la perspectiva de los directivos, docentes y alumnos de esas comunidades escolares? Y, adicionalmente, ¿qué recomendaciones pueden derivarse del análisis situado de la violencia escolar para la formulación de programas de intervención para la promoción de escuelas seguras?

La investigación se circunscribe a escuelas de Michoacán y Nuevo León, elección que responde al interés de observar el ambiente escolar en regiones del país que, particularmente a partir del 2008, experimentaron una notable incidencia de la inseguridad ciudadana asociada al narcotráfico y que, por tanto, son más vulnerables a padecer la violencia social que rodea y permea a las escuelas (INEGI, 2016). 


\section{Método}

Se adoptó un enfoque mixto CUAN + CUAL (Creswell, 2007) de tipo convergente paralelo. El apartado cuantitativo y el cualitativo se consideraron con el mismo valor en la fase de análisis, con un diagnóstico cuantitativo del ambiente escolar en torno a seis dimensiones y un análisis cualitativo fenomenológico.

Рara el análisis cuantitativo se aplicó el cuestionario de convivencia escolar directa mediante un blog creado para tal efecto (http://proyectoconacytescuelasegura.blogspot.mx/) utilizando el equipo de cómputo disponible en cada institución. Los grupos participantes fueron definidos de forma aleatoria de acuerdo al horario de clases de computación por los docentes de las instituciones. Para el análisis cualitativo se utilizó la entrevista semiestructurada aplicada a una muestra aleatoria de alumnos (dos de cada centro), así como a dos docentes y al director de cada plantel, con la finalidad de conocer su sentir respecto al ambiente escolar de su escuela y al programa en específico.

Durante el ciclo escolar 2015-2016 se eligieron diez secundarias públicas de los estados de Michoacán y Nuevo León (cinco por estado), concretamente en la ciudad de Morelia y en la zona metropolitana de Monterrey, con el criterio de que hubieran sido beneficiarias del apoyo del programa Es. Posteriormente se realizó un muestreo socioeconómico estratificado para incluir escuelas en zonas de clase media y zonas marginadas. El número total de estudiantes correspondiente a los tres grados de secundaria que participaron en el estudio cuantitativo fue de 1,950, mientras que para la muestra del cualitativo fue de 50 participantes.

Para el estudio cuantitativo se aplicó el cuestionario de convivencia escolar directa (Ortega-Ruiz, Del Rey y Casas, 2012) generado en el laboratorio de Violencia Escolar de la Universidad de Sevilla, compuesto por 37 reactivos en escala de Likert en torno a seis dimensiones:

1) Gestión de la red interpersonal: comprende las iniciativas de los docentes y padres de familia encaminadas a la convivencia dentro de la escuela.

2) Ajuste social entre iguales: se refiere a actitudes como amistad, cooperación y aceptación, así como el comportamiento de los jóvenes respecto a sus pares en la resolución de conflictos.

3) Ajuste a la disciplina democrática: la conducta del joven respecto a normas básicas de convivencia y las reglas de la escuela.

4) Victimización de iguales: las experiencias de bullying que han tenido los jóvenes.

5) Violencia ejercida por profesores: si es que se ha dado el caso abarcando conductas verbales, físicas y psicológicas.

6) Comportamiento violento: se refiere a la agresión hacia otras personas por parte del participante, sea de tipo físico, psicológico o de exclusión social.

En el análisis cualitativo el guión de las entrevistas se organizó en torno a los seis dominios del cuestionario para facilitar la discusión de los resultados de ambas evaluaciones. Para referenciar la fuente de la entrevista se usó el siguiente código: el estado (Mich=Michoacán; NL=Nuevo León), el número de la escuela según el registro y la inicial del rol del participante ( $D$ =directivo; $M=$ maestro/docente; y $\mathrm{N}=$ niño/alumno).

El procesamiento de los datos del cuestionario se realizó con SPSS versión 20 e incluyó: a) estadística descriptiva para diagnosticar la gravedad de la problemática en la convivencia escolar; b) fiabilidad (Alfa de Cronbach) y c) correlaciones de Pearson para reconocer relaciones significativas entre variables.

Para el análisis cualitativo de las entrevistas se utilizó el programa Atlas.ti (versión 7) según los principios de la Teoría Fundamentada (Glaser, 2001), sin imponer categorías de interpretación previas para rescatar 
así el sentido de la experiencia desde la perspectiva de sus participantes.

La integración de los dos procesos de análisis siguió cuatro etapas: 1) cada análisis se realizó de forma autónoma; 2) se discutió una primera interpretación compartida de los resultados cuantitativos y cualitativos; 3) cada línea de análisis volvió a trabajarse por separado con un nivel de sistematicidad mayor, y 4) se integraron y discutieron nuevamente los resultados (ver figura 1).

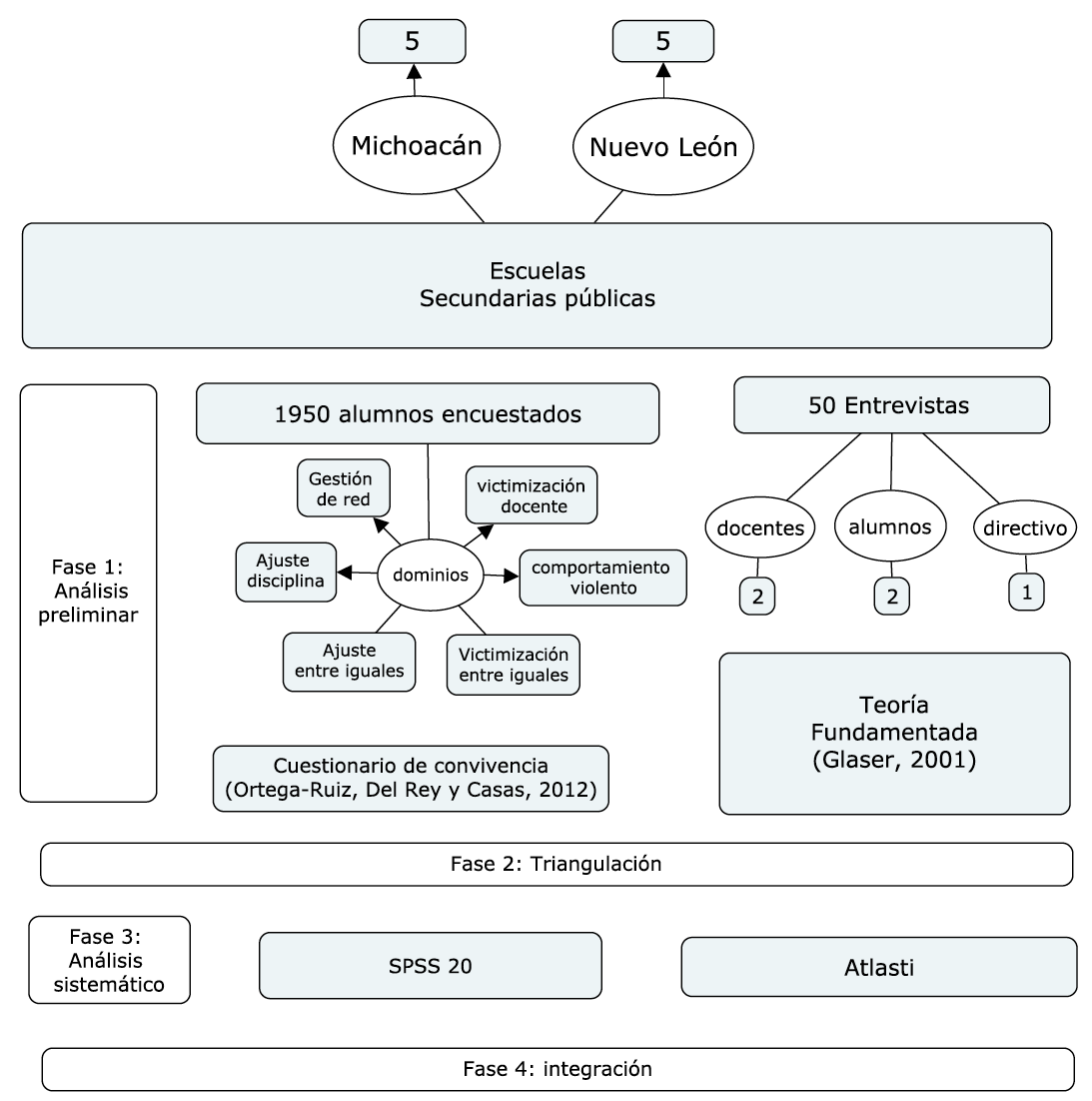

Figura 1. Esquema general de la metodología

\section{Resultados}

\subsection{Diagnóstico general de la convivencia escolar}

La estadística descriptiva de los cuestionarios (ver tabla 1) arroja un comportamiento positivo general en las diferentes dimensiones de la convivencia escolar. En casi todos los casos el puntaje promedio es significativamente superior a la media de esa dimensión, lo que significaría que los alumnos colaboran entre ellos para resolver sus conflictos (ajuste social entre iguales), con 24.14 de 28 posibles; que están familiarizados y respetan las normas básicas de convivencia en la escuela (ajuste a la disciplina democrática), con 23.23 de 28 posibles; que sus actos de bullying son infrecuentes (comportamiento violento), con sólo 2.37 sobre 16 puntos máximos de violencia; y que los profesores no contribuyen a generar un ambiente de convivencia negativo con los alumnos (victimización por el profesorado), 0.562 sobre 12. La dimensión en la que la media se aleja significativamente del extremo deseable es la gestión de la red interpersonal, relacionada con la participación de padres de familia y docentes. 
Tabla I. Puntajes promedio en cada las dimensiones del cuestionario

\begin{tabular}{l|c|c|c|c}
\hline Resultados generales & Media & $\begin{array}{l}\text { Máximo } \\
\text { puntaje } \\
\text { posible }\end{array}$ & $\begin{array}{c}\text { Máximos } \\
\text { obtenidos }\end{array}$ & $\begin{array}{c}\text { \% del puntaje } \\
\text { máximo posible }\end{array}$ \\
\hline Gestión de la red interpersonal & 22.88 & 36 & 36 & 63.50 \\
Ajuste social entre iguales & 24.14 & 28 & 28 & 86.21 \\
Victimización de iguales & 2.98 & 24 & 24 & 12.41 \\
Victimización por profesorado & .56 & 12 & 11 & 5.10 \\
Ajuste a la disciplina democrática & 23.23 & 28 & 28 & 88.07 \\
Comportamiento violento & 2.37 & 16 & 16 & 14.81 \\
\hline
\end{tabular}

Estos datos coinciden en términos generales con los obtenidos en los informes estatales de Michoacán y Nuevo León (SEP, 2015), en los que la prevalencia de la violencia es menor de la que cabría esperar al tenor de estudios internacionales (OCDE, 2013) que sitúan a México entre los primeros lugares con mayor incidencia de acoso escolar. Sin embargo, el análisis cualitativo de las entrevistas problematiza ese panorama, revelando una tendencia a la ocultación de los problemas de convivencia ante la evaluación de los observadores. Se da, además, en los tres agentes escolares clave: a) en los alumnos, en quienes predomina la inhibición (titubean, responden afirmativamente a algunas preguntas sin entenderlas bien); b) en los docentes, que tienden a minimizar los problemas de convivencia y a resaltar sus capacidades pedagógicas para incentivar un buen ambiente escolar, situándose meramente como testigos o como autoridades, es decir, como agentes externos al problema y no como sujetos que forman parte de él; y c) en los directivos, los cuales orientan su testimonio a presentar un panorama positivo de convivencia, resaltando las políticas, protocolos y programas llevados a cabo (sin definir con claridad y precisión qué hicieron con los fondos del programa ES).

Esta actitud supone un sesgo positivo en la percepción de la convivencia y contribuye a hacer de la escuela una especie de "caja negra". Por ello, el análisis de las entrevistas fue determinante para tener un diagnóstico más integral sobre la convivencia al interior de las escuelas. A continuación se exponen los resultados en torno a las dimensiones claves del estudio:

Gestión de la red interpersonal. Son recurrentes los testimonios de docentes que denuncian que no cuentan con el suficiente apoyo por parte de los padres, a quienes responsabilizan en gran medida del origen de los problemas de convivencia que se dan en la escuela (la tabla II se ofrecen dos testimonios).

Tabla II. Testimonios de docentes acerca de la responsabilidad de los padres en los comportamientos no deseados de los alumnos en la escuela

\begin{tabular}{l|l|l}
\hline Testimonio & Implicaciones & Informante \\
\hline $\begin{array}{l}\text { [los padres] se desatienden mucho de los niños, a veces se les cita a } \\
\text { reunión, y tenemos un grupo de } 40 \text { y sólo tenemos 20. [...]. }\end{array}$ & $\begin{array}{l}\text { Falta de cooperación } \\
\text { entre padres y docentes. }\end{array}$ & Mich114M1 \\
$\begin{array}{l}\text { También se necesitaría trabajar quizá con los padres de familia, lo } \\
\text { malo es que ellos luego no cooperan, nos da la impresión que } \\
\text { sienten como que aquí es la guardería. }\end{array}$ & \\
$\begin{array}{l}\text { Ahora con esta reforma nos dicen que somos tres los actores, pero } \\
\text { de repente a nosotros nos falla el tercero, nos falta una escuela de } \\
\text { padres, es decir, necesito que estas dos semanas estén, necesito } \\
\text { que estas dos semanas nos ayuden, que se involucren más. }\end{array}$ & $\begin{array}{l}\text { Distancia entre el } \\
\text { discurso oficial de } \\
\text { integración y la realidad } \\
\text { (padres ausentes). }\end{array}$ & MtyS29M2 \\
\hline
\end{tabular}

La visita de los padres a la escuela en muchas ocasiones responde a que su hijo ha contravenido alguna regla o presenta un mal desempeño académico. Por lo tanto, suele estar asociada a algún mal, es generadora de estrés y, en definitiva, no está dirigida a su participación en la prevención de los conflictos ni en la construcción de dinámicas educativas positivas de convivencia. 
Ajuste a la disciplina democrática. Las entrevistas confirman lo revelado por los cuestionarios. Los alumnos reportan ser conscientes del comportamiento no aceptable en la escuela. Las prácticas de vigilancia y control por parte de maestros, prefectos y directivos contribuyen a reforzar esa percepción compartida de que dentro de la escuela (especialmente en el aula) no están permitidas las manifestaciones de violencia entre los alumnos. No obstante, esa norma no parece ser fruto del consenso de la comunidad. Se entiende como una norma establecida desde fuera, incuestionable, no situada, no negociada, como ilustra el siguiente testimonio de un directivo:

Primero se habla con los padres de familia cuando se les entrega el reglamento, a la hora de distribuirse, se les entrega y se dice el alumno tiene que cumplir con estos puntos, lo que debe hacer, y más que todo lo que no debe de hacer, ¿verdad?; entonces, si infringes ciertas reglas va a haber una sanción, entonces ellos ya saben que lo tienen que cumplir para permanecer aquí en la escuela (NL29D).

Las normas están orientadas a evitar y sancionar los actos que las transgreden y no a la construcción de valores positivos de convivencia. Cuando se pregunta a los alumnos si consideran que su comportamiento es bueno formulan su respuesta a la inversa, con más conciencia del mal que de la virtud: "Sí... porque no soy irrespetuoso, ni nada por el estilo, malo" (NL100N1); "Yo no digo groserías, me porto bien" (Mich100N2).

Esa moral heterónoma (Piaget, 1983) favorece que la conducta no deseable se manifieste en comportamientos más difíciles de sancionar, como robos anónimos o "desapariciones" de objetos. O bien, se eligen espacios escolares menos controlados (como el patio o los baños) como escenarios de diferentes tipos de violencia (peleas, coacciones, abuso de poder, entre otros).

Ajuste social entre iguales. Cuando se les pregunta a los alumnos cómo reaccionan cuando son testigos de violencia o abuso por parte de algunos niños hacia otros las respuestas oscilan entre la intervención para defender a la víctima, la denuncia a las autoridades escolares y la pasividad. En ningún caso pudo atestiguarse la existencia de iniciativas de solución dialogada del conflicto entre los protagonistas. Tampoco hay testimonios de que en el aula se construya algún tipo de participación de los alumnos en la definición de las normas y sanciones.

En cambio, sí reportan la existencia de grupos de poder entre los propios alumnos, normalmente en relaciones de rivalidad o discriminación hacia otros grupos dentro de la escuela o el aula. En este sentido, la justicia entre iguales estaría dirimida por relaciones de poder entre grupos. Este ecosistema moral es poco propicio para generar confianza futura en las instituciones democráticas de justicia y seguridad como reguladores de conducta social.

Victimización entre iguales. La mayor parte de los alumnos no confiesa en sus entrevistas haber sido víctima de agresión, ni siquiera objeto de burla o rechazo. Lo más frecuente es que se presenten como espectadores de la violencia que otros sufren. No obstante, sí hay ejemplos ilustrativos de su condición eventual de víctimas, especialmente de un tipo de problema en la convivencia que pasa fácilmente desapercibido en evaluaciones externas al no tener una manifestación violenta pero que, a juicio de los entrevistados, causa mucho sufrimiento: el de la exclusión. Se trata de una violencia silenciosa que tiene como consecuencia el rechazo de un niño en el grupo o la clase.

La tabla III expone la experiencia de dos alumnos de la misma escuela (víctimas de exclusión) que interpretan su experiencia de forma muy diferente: mientras la alumna 1 parte de una concepción normativa de la moral y pide más protección y control, el alumno 2 parte de una visión más democrática y desea un mayor diálogo y oportunidades para la convivencia. 
Tabla III. Casos de victimización infantil que representan dos modelos de convivencia escolar

\begin{tabular}{|c|c|c|c|c|}
\hline & \multicolumn{2}{|c|}{ Concepción normativa } & \multicolumn{2}{|c|}{ Concepción democrática } \\
\hline & \multicolumn{2}{|c|}{ Caso: alumna 1} & \multicolumn{2}{|c|}{ Caso: alumno 2} \\
\hline \multirow{4}{*}{$\begin{array}{l}\text { Modelo de } \\
\text { convivencia }\end{array}$} & $\begin{array}{l}\text { Razón de la } \\
\text { victimización }\end{array}$ & Testimonio & $\begin{array}{l}\text { Razón de la } \\
\text { victimización }\end{array}$ & Testimonio \\
\hline & Físico: Tamaño & $\begin{array}{l}\text { "los niños de taller de } \\
\text { Electrónica me decían, tu } \\
\text { no debes estar aquí } \\
\text { porque tú estás muy } \\
\text { chiquita, pero sí tengo los } \\
12 \text { años" }\end{array}$ & $\begin{array}{l}\text { Gustos } \\
\text { diferentes }\end{array}$ & $\begin{array}{l}\text { "Desde que entré a primero } \\
\text { [...] cuando me quería juntar } \\
\text { con algunos, pues yo les } \\
\text { platicaba cosas, por ejemplo } \\
\text { qué música me gustaba [...] } \\
\text { y a ellos no les gustaba, eran } \\
\text { diferentes a mí y por eso } \\
\text { pienso que me molestan" }\end{array}$ \\
\hline & $\begin{array}{c}\text { Factor clave } \\
\text { para la buena } \\
\text { convivencia }\end{array}$ & Testimonio & $\begin{array}{l}\text { Factor clave } \\
\text { para la buena } \\
\text { convivencia }\end{array}$ & Testimonio \\
\hline & La obediencia & $\begin{array}{l}\text { "Que las niñas de esta } \\
\text { escuela se porten bien, } \\
\text { obedezcan a sus maestros, } \\
\text { no traten de buscar peleas, } \\
\text { estudien y trabajen, no } \\
\text { vengan nada más a perder } \\
\text { su tiempo". }\end{array}$ & El diálogo & $\begin{array}{l}\text { "Que los maestros que } \\
\text { trabajen en la escuela hablen } \\
\text { más con los alumnos, que } \\
\text { convivamos más, porque la } \\
\text { convivencia hace que te } \\
\text { lleves bien con los demás". }\end{array}$ \\
\hline \multirow[t]{2}{*}{ Soluciones } & Más "comandos" & Beneficio & \multirow[t]{2}{*}{ Más "convivios" } & Beneficio \\
\hline & & $\begin{array}{l}\text { Para aumentar la } \\
\text { protección dentro de la } \\
\text { escuela }\end{array}$ & & $\begin{array}{l}\text { Para que padres, niños y } \\
\text { maestros puedan convivir de } \\
\text { manera más armónica }\end{array}$ \\
\hline
\end{tabular}


Comportamiento violento. Si nos apegamos a los datos obtenidos en los cuestionarios, el diagnóstico sobre el grado de violencia escolar puede ser engañosamente benigno; sin embargo, las entrevistas dan una idea de la complejidad del fenómeno y de la diversidad de formas y razones que puede asumir el comportamiento violento al interior de la escuela (figura 2).

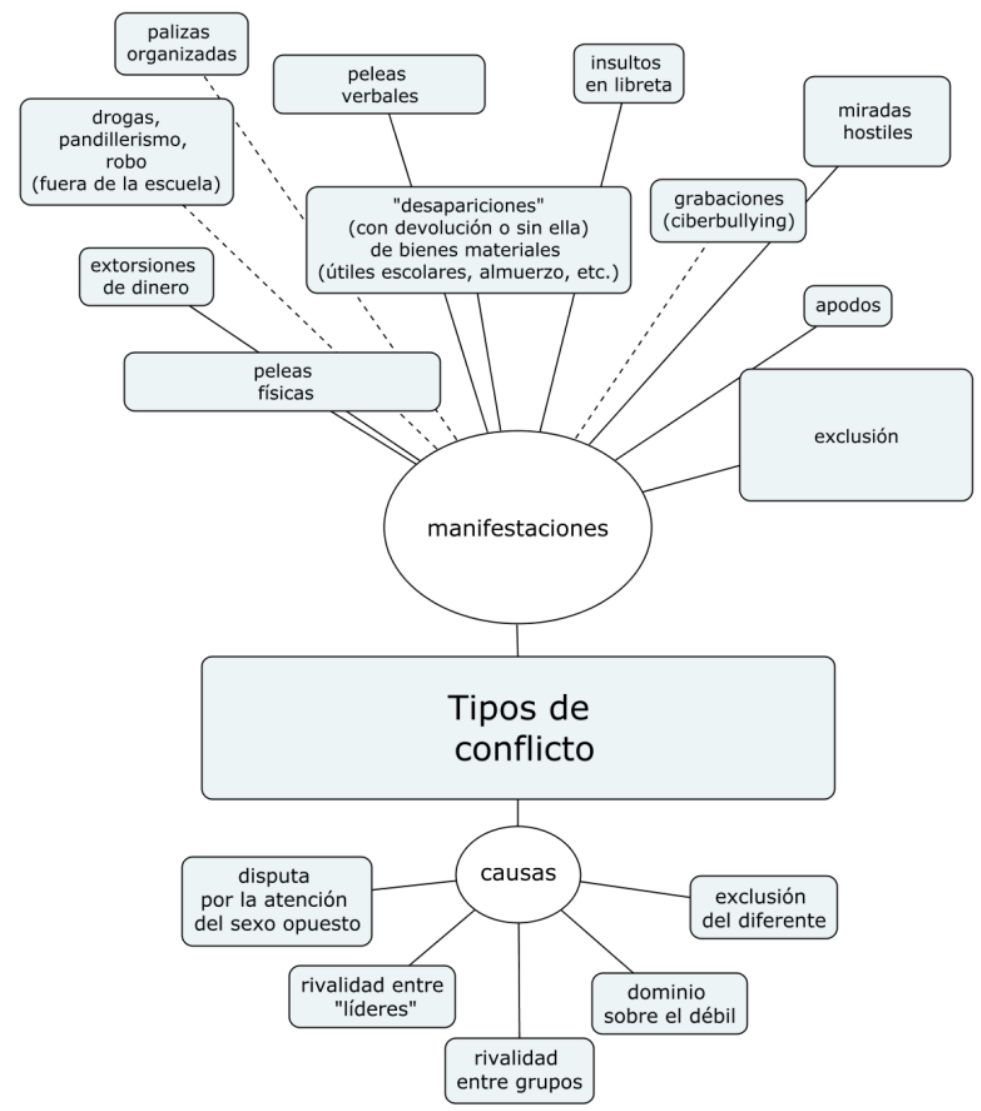

Figura 2. Manifestaciones de la violencia en las escuelas, según entrevistas analizadas

Los participantes refieren explícitamente una diversidad de detonantes de la violencia; en algunos casos la atribuyen a la necesidad de dominio de unos alumnos sobre otros, ya sea para obtener algo a cambio o para demostrar su poder. Varios testimonios revelan la existencia de grupos enfrentados de alumnos (a veces también de maestros) que construyen identidades compartidas y sentidos de pertenencia para definir su legitimidad frente a los otros grupos. Esta cultura hace de la escuela un espacio de disputa por el poder y, en última instancia, supone la normalización de un pandillerismo incipiente. Así queda atestiguado incluso en el lenguaje que se usa eventualmente en las entrevistas, el cual remite a un contexto social de lucha: "Soy el jefe del grupo" (Mich114N1); "los comandos" (Mich100N2).

Victimización por parte de los docentes. En general, los alumnos reportan en las entrevistas una imagen positiva de los profesores, ya sea como garantes del orden y la disciplina (y, por tanto, sancionadores de conductas inapropiadas) o bien por haber recibido su apoyo en una situación emocional problemática.

No obstante, cabe matizar esa percepción. Según esos testimonios, habría dos tipos de docentes: Los que se dedican a impartir su materia y no se involucran en la construcción de un ambiente escolar saludable (a quienes los alumnos entrevistados perciben de forma negativa, como cómplices por su pasividad de los juegos de poder abusivos de unos alumnos frente a otros), el siguiente testimonio refleja la valoración que hace un alumno de ese perfil docente: 
[lo más valioso sería que los profesores aprendieran a] escuchar cuando un alumno les dice algo, porque hay unos maestros que no les importa, que ellos vienen nada más a trabajar y se van, pero para mí, ser maestro es enseñarles un buen camino a los alumnos. (Mich100N1).

El segundo perfil de docente,es el de aquellos que generan relaciones de confianza con los alumnos, muestran disposición a escucharlos y los asesoran con sus problemas de confianza o aislamiento; los maestros con este perfil son percibidos con agradecimiento. El siguiente testimonio de un docente refleja esa actitud más dialógica:

Primero es el diálogo, escuchar ambas partes, siempre se les tiene que dar la oportunidad a cada uno, la misma oportunidad, porque nunca sabemos cuál comenzó o por qué, [...] más que nada es darles la oportunidad de explicar qué sucedió, hablar con los padres y llegar a un acuerdo. (NL100M2).

Las diferencias entre ambos tipos de docentes pueden ser interpretadas como un contraste entre una actitud monológica y una más dialógica. En una actitud monológica hay una sola voz distante, en una actitud dialógica hay una comunicación más plural e integradora del otro (Fernández-Cárdenas, 2014).

\subsection{Relación entre las dimensiones de la violencia escolar}

Por último, es importante destacar que el instrumento aplicado para la observación de la violencia escolar (Ortega-Ruiz, Del Rey y Casas, 2012) demostró su confiablidad en el contexto escolar bajo estudio, aunque con un índice de fiabilidad ligeramente menor en el contexto mexicano y, en consecuencia, hace repensar la conveniencia de una adaptación del instrumento a las condiciones locales.

El instrumento ofrece la posibilidad de reconocer correlaciones especialmente significativas entre las dimensiones de la violencia escolar. La aplicación de correlaciones de Pearson entre variables arroja una fuerte correlación de carácter negativo entre la gestión de la red interpersonal y la victimización de iguales, es decir, entre la falta de implicación de los padres de familia en la escuela y la manifestación de la violencia entre los alumnos dentro de ella (ver tabla IV).

Tabla IV. Correlación de Pearson entre dimensiones de la violencia escolar

\begin{tabular}{l|l|c|c}
\hline \multicolumn{2}{c|}{ Dimensiones } & $\begin{array}{c}\text { Gestión } \\
\text { de la red }\end{array}$ & $\begin{array}{c}\text { Victimización } \\
\text { de iguales }\end{array}$ \\
\hline \multirow{2}{*}{$\begin{array}{l}\text { Gestión de la red } \\
\text { interpersonal }\end{array}$} & Correlación de Pearson & 1 & $-.291^{* *}$ \\
\cline { 2 - 4 } & Sig. (bilateral) & & .000 \\
\cline { 2 - 4 } & $\mathrm{N}$ & 1950 & 1950 \\
\hline \multirow{2}{*}{$\begin{array}{l}\text { Victimización de } \\
\text { iguales }\end{array}$} & Correlación de Pearson & $-.291^{* *}$ & 1 \\
\cline { 2 - 4 } & Sig. (bilateral) & .000 & 1950 \\
\cline { 2 - 4 } & $\mathrm{N}$ & 1950 & \\
\hline
\end{tabular}

**La correlación es significativa en el nivel 0.01 (2 colas).

\subsection{Visibilización del impacto del programa ES}

La visibilidad de ES al interior de las escuelas, a juicio de los participantes, no fue amplia. Excepto los directivos, la mayoría de los docentes y, sin excepción, todos los alumnos entrevistados, no reportaron tener conocimiento de las acciones en su centro derivadas de este programa, como ilustra el testimonio de este alumno: "Nada más nos mencionó que el programa de las escuelas seguras estaba aprendiendo algo, pero no alcancé a escuchar bien" (Mich114N1).

En el caso de los docentes, la idea que tienen de es suele ser difusa y señalaron en varias ocasiones que sienten que es algo que atañe a un docente específico de ética o ciudadanía. Y lo común en los directivos es reportar acciones emprendidas en su escuela bajo el estímulo de ES pero, al mismo tiempo, señalan falta de apoyo y seguimiento, y el alcance limitado del programa en la práctica. 
Por otro lado, los participantes relacionan el programa fundamentalmente con dos tipos de acciones:

1) Reforzamiento de las medidas de protección, como en el caso de la compra de cámaras de vigilancia (NL29D).

2) Sensibilización de la comunidad escolar a través del discurso. Es el caso de las pláticas (Mich100M1) o cursos (Mich108M1) ofrecidos por expertos invitados por la escuela, así como la disponibilidad de documentos con el contenido del programa ES (NL29D).

\section{Conclusiones}

Los resultados deben limitarse a los contextos escolares observados, ya que la muestra no permite generalizarlos al conjunto país. No obstante, creemos que una ventaja de la metodología mixta es obtener un diagnóstico situado que, por una parte, está sustentado en evidencia estadística en torno a diferentes dimensiones (y su relación entre ellas) y, por otra, es capaz de acceder a la percepción interna de la escuela respecto a su clima de convivencia. El diagnóstico revela la falta de pluralidad de perspectivas de los participantes en la conformación de una comunidad escolar con mecanismos de regulación social basados en el diálogo y la participación.

En las comunidades educativas analizadas predomina la percepción de que la escuela tiene entre sus funciones principales vigilar, controlar y penalizar las "malas conductas". Subyace la metáfora de la escuela como centro penitenciario o guardería y no como institución en la cual construir una cultura de solidaridad y colaboración. Asimismo, la violencia escolar se concibe como algo asociado a ciertos individuos o a conductas, por lo que la solución a los problemas de convivencia implicaría la expulsión o sanción a aquellos que la infringen, sin que eso signifique un cambio más profundo en las normas de convivencia, en los modelos pedagógicos o en el desarrollo moral de la comunidad escolar entera.

Tabla V. Modelos de intervención en virtud de la concepción de la violencia escolar

\begin{tabular}{c|l|l|l}
\hline $\begin{array}{c}\text { Origen } \\
\text { de la violencia }\end{array}$ & \multicolumn{1}{|c|}{$\begin{array}{c}\text { Manifestación } \\
\text { de la violencia }\end{array}$} & \multicolumn{1}{c}{$\begin{array}{c}\text { Modelo de concepción } \\
\text { del fenómeno }\end{array}$} & \multicolumn{1}{c}{ Política de intervención } \\
\hline Individual & $\begin{array}{l}\text { Conductas violentas } \\
\text { o delictivas. }\end{array}$ & $\begin{array}{l}\text { Monológico: basado en la } \\
\text { instrucción. } \\
\text { Sedes relacionales y } \\
\text { Social y sistémico: basado en la } \\
\text { concepciones éticas. }\end{array}$ & $\begin{array}{l}\text { Refuerzo de un sistema escolar de } \\
\text { control, vigilancia y penalización. } \\
\text { concientización y educación ética } \\
\text { de toda la comunidad escolar. }\end{array}$ \\
\hline
\end{tabular}

La tabla $V$ contrasta dos concepciones de la violencia asociadas a dos modelos de intervención. Las entrevistas documentan en los participantes (directivos, docentes $y$, alumnos) el predominio de la concepción individual y conductual de la violencia escolar y, por tanto, la aceptación implícita de un modelo de intervención monológico basado en el control, al cual de hecho responde el modelo del programa ES. Sin embargo, esas políticas, a pesar del beneficio de inhibir la manifestación de la violencia dentro de la escuela, no generan la construcción a largo plazo de un ambiente escolar de convivencia positiva.

Nos sumamos, por tanto, a quienes defienden modelos de intervención dialógicos (Fernández-Cárdenas, 2014), que toman en cuenta a los participantes como sujetos activos en su construcción y desarrollo (Furlán Malamud, 2012; Olweus, 2004; Van Dijk, 2012; Viguer y Avià, 2009). En palabras de Ortega et al. (2005, p. 164) las intervenciones documentadas más exitosas están basadas en el involucramiento y participación de la comunidad educativa: "las comunidades discuten y pactan reglas; estudiantes, padres y profesores trabajan en equipo".

El programa es ha finalizado su período de aplicación, dando paso a nuevas iniciativas nacionales, como el Programa Nacional de Convivencia Escolar (PNCE), el cual trasciende el enfoque monológico del programa ES, centrado en exceso en el problema de la seguridad física, y apunta a la construcción social y situada de la convivencia escolar (Chávez, Gómez-Nashiki, Ochoa y Zurita, 2016). 
En sintonía con la perspectiva dialógica de esta investigación, a manera de colofón hacemos una síntesis de recomendaciones para la fundamentación de programas orientados a formar escuelas seguras:

1) Fortalecer la comunicación de las acciones de los programas dentro de las escuelas como parte integral de la agenda, así como el conocimiento y discusión de los mismos participantes de los efectos concretos en la mejora de su convivencia.

2) Implementar talleres y actividades de visibilización de a) la violencia en todas sus expresiones, especialmente de aquellos tipos de violencia aceptados como legítimos o naturalizados por su carácter habitual; y b) las dinámicas que se dan entre bullies, víctimas y espectadores, para que los participantes de la comunidad escolar puedan reinterpretar estos roles y cobrar conciencia de las funciones que cumplen en el reconocimiento social.

3) Involucrar de manera más participativa a padres de familia, no sólo a través de reuniones informativas, sino también de consejos de acción participativa con una mayor injerencia en las estrategias implementadas en las escuelas.

4) Capacitar a profesores en la modelación de prácticas curriculares y pedagógicas que transciendan el paradigma de la transmisión de valores o los modelos normativos-sancionadores para impulsar la construcción de ambientes de convivencia basados en el aprendizaje colaborativo y la participación de toda la comunidad.

\section{Agradecimiento}

Esta investigación deriva del proyecto "Estudio Comparativo sobre resultados del programa Escuela Segura en los Estados de Michoacán y Nuevo León", financiado por el proyecto CONACYT SEP/SEB$2014 / 239204$.

\section{Referencias}

Auditoría Superior de la Federación (2013). Informe del Resultado de la Fiscalización Superior de la Cuenta Pública 2011 [Informe ejecutivo]. Cámara de Diputados, México. Recuperado de http://www.asf.gob.mx/trans/informes/ir2011i/Indice/Ejecutivo.htm

Creswell, J. W. (2007). Designing and conducting mixed methods research. Thousand Oaks, CA: Sage.

Chávez, C., Gómez-Nashiki, A., Ochoa, A. y Zurita, C. (2016). La política nacional de convivencia escolar de México y su impacto en la vida en las escuelas de educación básica. Revista Posgrado y Sociedad, 14(1), 113.

Fernández-Cárdenas, J. M. (2014). El dialogismo: secuencialidad, posicionamiento, pluralidad e historicidad en el análisis de la práctica educativa. Sinéctica, 43, 183-203.

https://sinectica.iteso.mx/index.php/SINECTICA/article/view/17

Fierro, C., Lizardi, A., Tapia, G. y Juárez, M. (2013). Convivencia escolar. Un tema emergente de investigación educativa en México. En A. Furlán y T. Spitzer (Coords.), Violencia en las escuelas. Problemas de convivencia y disciplina. México: COMIE/ ANUIES.

Furlán, A. (2012). Inseguridad y violencia en la educación. Problemas y alternativas. Perfiles Educativos, 34, 118-128. Recuperado de http://www.iisue.unam.mx/perfiles/articulo/2012-e-inseguridad-y-violencia-enla-educacion-problemas-y-alternativas.pdf 
Furlán, A. y Saucedo, C. (2008). Violencia en la escuela: las perspectivas culturales de los actores sociales en contextos situados de práctica. Informe de Investigaciones Educativas, 22(2), 231-245.

Furlán, A. y Spitzer, T. (Coords.). (2013). Violencia en las escuelas. Problemas de convivencia y disciplina. México: COMIE/ANUIES.

Glaser, B. G. (2001). The grounded theory perspective: conceptualization contrasted with description. Mill Valley, cA: Sociology Press.

Gómez-Nashiki, A. (2014). La violencia de alumnos hacia maestros en escuelas secundarias de Colima. Revista de Investigación Educacional Latinoamericana, 51(2), 19-34.

Instituto Nacional de Estadística y Geografía. (2016). Encuesta Nacional de Victimización y Percepción sobre Seguridad Pública (ENVIPE). México: Autor.

Klein, J., Cornell, D. y Konold, T. (2012). Relationships between bullying, school climate, and student risk behaviors. School Psychology Quarterly, 27(3), 154-169. doi:10.1037/a0029350

Nickerson, A. B., Singleton, D., Schnurr, B. y Collen, M. H. (2014). Perceptions of school climate as a function of bullying involvement. Journal of Applied School Psychology, 30(2), 157-181.

OCDE (2013). Teaching and Learning International Survey (TALIS 2013). Recuperado de http://www.oecd.org/edu/school/talis.htm

Olweus, D. (2004). Conductas de acoso y amenaza entre escolares. Madrid: Morata.

Ortega, S., Ramírez, M. y Castelán, A. (2005). Estrategias para prevenir y atender el maltrato, la violencia y las adicciones en las escuelas públicas de la Ciudad de México. Revista Iberoamericana de Educación, 38, 147-169. Recuperado de http://rieoei.org/rie38.htm

Ortega-Ruiz, R., Del Rey, R. y Casas, J. A. (2012). La convivencia escolar: clave en la predicción del bullying. Revista Iberoamericana de Evaluación Educativa, 6(2), 91-102. Recuperado de http://www.rinace.net/riee/numeros/vol6-num2/art04.pdf

Piaget, J. (1983). El criterio moral del niño. Barcelona: Fontanella.

Secretaría de Educación Pública. (2015). Diagnóstico ampliado. Programa Nacional de Convivencia Escolar. Recuperado de https://www.gob.mx/cms/uploads/attachment/file/147558/Diagnostico Ampliado.pdf

Viguer, P. y Avià, S. (2009). Un modelo local para la promoción de la convivencia y la prevención de la violencia entre iguales desde el ámbito comunitario. Cultura y Educación, 21(3), 345-349.

Van Dijk, S. (2012). La política pública para abatir el abandono escolar y las voces de los niños, sus tutores y sus maestros. Revista Mexicana de Investigación Educativa, 17(52), 115-139. Recuperado de http://www.comie.org.mx/v1/revista/portal.php?idm=es\&sec=SC03\&\&sub=SBB\&criterio=ART52005

Vega, M. G. y González, G. J. (2016). Bullying en la escuela secundaria. Factores que disuaden o refuerzan el comportamiento agresor de los adolescentes. Revista Mexicana de Investigación Educativa, 21(71), 11651189. Obtenido de https://www.comie.org.mx/v1/revista/portal.php?idm=es\&sec=SC03\&\&sub=SBB\& criterio=ART71007

Zorrilla, M. Z. (Coord.) (2008). Informe final de la evaluación del diseño del Programa Nacional de Escuela Segura. Universidad Autónoma de Aguascalientes. 
Zurita, U. (2016). La violencia escolar desde los medios de comunicación y el poder legislativo en México. Sophia, 11(1), 81-93. Recuperado de http://revistas.ugca.edu.co/index.php/sophia/article/view/301 